\title{
Metallic and superconducting gallane under high pressure
}

\author{
Guoying Gao, ${ }^{1}$ Hui Wang, ${ }^{1}$ Aitor Bergara,,${ }^{2,3,4}$ Yinwei Li, ${ }^{1}$ Guangtao Liu, ${ }^{1}$ and Yanming $\mathrm{Ma}^{1},{ }^{1,}$ \\ ${ }^{1}$ State Key Lab of Superhard Materials, Jilin University, Changchun 130012, P. R. China \\ ${ }^{2}$ Materia Kondentsatuaren Fisika Saila, Zientzia eta Teknologia Fakultatea, Euskal Herriko Unibertsitatea, \\ 644 Postakutxatila, 48080 Bilbo, Basque Country, Spain \\ ${ }^{3}$ Donostia International Physics Center (DIPC), Paseo de Manuel Lardizabal, 20018, Donostia, Basque Country, Spain \\ ${ }^{4}$ Centro de Fisica de Materiales CSIC-UPVIEHU, 1072 Posta kutxatila, E-20080 Donostia, Basque Country, Spain
}

(Received 15 May 2011; revised manuscript received 6 July 2011; published 24 August 2011)

\begin{abstract}
Using our newly developed particle swarm optimization algorithm on crystal structural prediction, we characterized the pressure-induced structural transition sequence of gallane $\left(\mathrm{GaH}_{3}\right)$. As has been observed in alane $\left(\mathrm{AlH}_{3}\right)$, enthalpy calculations reveal that the $P m \overline{3} n$ structure of $\mathrm{GaH}_{3}$ becomes stable above $160 \mathrm{GPa}$, below which it is unstable with respect to elemental decomposition. Interestingly, the $P m \overline{3} n$ structure is metallic, and the application of the Allen-Dynes modified McMillan equation reveals a high superconducting transition temperature $\left(T_{\mathrm{c}}\right)$, which reaches $86 \mathrm{~K}$ at $160 \mathrm{GPa}$ and increases with decreasing pressure $\left(T_{c}=102 \mathrm{~K}\right.$ at $120 \mathrm{GPa}$ ). Our band structure calculations demonstrate that $\mathrm{GaH}_{3}$ within the $P m \overline{3} n$ structure is a highly ionic solid, where the ionicity of $\mathrm{H}$ atoms plays an important role in the predicted high temperature superconductivity.
\end{abstract}

DOI: 10.1103/PhysRevB.84.064118

\section{INTRODUCTION}

Long ago, Ashcroft predicted that metallic hydrogen might be a high-temperature superconductor, ${ }^{1}$ however, pressureinduced metallic transition of hydrogen is still an elusive goal of physics, even at pressures around $300 \mathrm{GPa}^{2-4}$ Recently, it has been proposed that hydrogen-rich compounds, such as group IVa hydrides $\left(\mathrm{SiH}_{4}, \mathrm{GeH}_{4}\right.$, and $\left.\mathrm{SnH}_{4}\right)$, might become metallic at lower pressures than hydrogen and might well be superconductors with high critical temperatures. ${ }^{5}$ Although the metallicity and superconductivity experimentally observed in $\mathrm{SiH}_{4}$ under pressure 6,7 were actually attributed to the formation of $\mathrm{PtH}$ and $\mathrm{Si}^{8,9}$ its metallization pressure was predicted to be lower than in solid hydrogen. ${ }^{10,11}$ Our previous work also suggested that $\mathrm{GeH}_{4}{ }^{12,13}$ and $\mathrm{SnH}_{4}{ }^{14}$ might metallize and become good superconductors at experimentally accessible pressures. These results are sufficiently encouraging to prompt further studies of a wider range of hydrides.

Due to its potential application as a hydrogen storage material, alane $\left(\mathrm{AlH}_{3}\right)$ has recently been the subject of many theoretical and experimental investigations. ${ }^{15,16}$ Using a "random searching" technique, Pickard et al. ${ }^{16}$ predicted a metallic cubic phase (space group $P m \overline{3} n$ ) for high-pressure $\mathrm{AlH}_{3}$. Subsequently, Goncharenko et al. ${ }^{15}$ indeed observed that $\mathrm{AlH}_{3}$ becomes metallic at $100 \mathrm{GPa}$ and confirmed the predicted structure. However, no superconducting transition was found down to $4 \mathrm{~K}$, which disagrees with the theoretically estimated $T_{c}$ of $\sim 20 \mathrm{~K} .{ }^{15}$ The origin for this disagreement between theory and experiment was explained by the high anharmonic renormalization of selected phonon modes inducing the superconducting transition, which considerably decreased the electron-phonon coupling parameter $\lambda .{ }^{17}$ Considering the larger polarizability of $\mathrm{GaH}_{3}$ compared to $\mathrm{AlH}_{3}$, according to the Goldhammer-Herzfeld criterion, ${ }^{18} \mathrm{GaH}_{3}$ should be a good candidate to metallize at even lower pressures. In this article, we apply our newly developed particle swarm optimization (PSO) technique on crystal structure prediction ${ }^{19}$ to extensively explore the crystal structures of $\mathrm{GaH}_{3}$ under high pressure and characterize the electronic, dynamical, and
PACS number(s): 74.70.Ad, 74.10.+v, 74.25.Jb, 74.62.Fj

superconducting properties of the predicted high-pressure structures.

\section{COMPUTATIONAL DETAILS}

The crystal structure prediction is based on a global minimization of free-energy surfaces merging ab initio totalenergy calculations via PSO technique as implemented in the Crystal structure AnaLYsis by Particle Swarm Optimization (CALYPSO) code. ${ }^{19}$ This methodology has been successfully applied to many high-pressure structures on elemental, binary, and ternary compounds with metallic, ionic, and covalent bondings. ${ }^{19-22}$ The underlying ab initio structural relaxations were performed using density functional theory within the Perdew-Burke-Ernzerh (PBE) parameterization of the generalized gradient approximation $(\mathrm{GGA})^{23}$ as implemented in the Vienna $a b$ initio Simulation Package (VASP) code. ${ }^{24}$ The all-electron projector-augmented wave (PAW) method $^{25}$ was adopted with the PAW potentials, where $1 s$ and $3 d 4 s 4 p$ are treated as valence electrons for $\mathrm{H}$ and $\mathrm{Ga}$ atoms, respectively. A plane-wave basis set with an energy cutoff of $800 \mathrm{eV}$ was used to get well-converged total energies. On the other hand, the plane-wave pseudopotential method within the PBE-GGA as implemented in the Quantum-ESPRESSO package ${ }^{26}$ was used to characterize the electronic properties, lattice dynamics, and electron-phonon coupling (EPC) for $P m \overline{3} n \mathrm{GaH}_{3}$. For these purposes, ultrasoft pseudopotentials for $\mathrm{H}$ and $\mathrm{Ga}$ were considered, and convergence tests concluded that suitable values would be a 100 Ry kinetic energy cutoff and a $12 \times$ $12 \times 12$ Monkhorst-Pack (MP) $)^{27} k$-point sampling mesh for the electronic Brillouin zone (BZ) integration. Phonon frequencies were calculated based on the density functional linear-response theory, ${ }^{28}$ and a $4 \times 4 \times 4 q$-mesh in the first $\mathrm{BZ}$ was used in the interpolation of the force constants for the phonon dispersions. The EPC spectral function $\alpha^{2} F(\omega)$ can be expressed in terms of the phonon linewidth $\gamma_{q j}$, owing to electron-phonon scattering, ${ }^{29-31}$

$$
\alpha^{2} F(\omega)=\frac{1}{2 \pi N_{f}} \sum_{q j} \frac{\gamma_{q j}}{\omega_{q j}} \delta\left(\hbar \omega-\hbar \omega_{q j}\right),
$$


where $N_{f}$ is the electronic density of states per atom and spin at the Fermi level. The linewidth of a phonon mode $j$ at wave vector $q, \gamma_{q j}$, arising from electron-phonon interaction is given by

$$
\gamma_{q j}=2 \pi \omega_{q j} \sum_{k n m}\left|g_{k n, k+q m}^{j}\right|^{2} \delta\left(\varepsilon_{k n}\right) \delta\left(\varepsilon_{k+q m}\right),
$$

where the sum is over the BZ, and $\epsilon_{k n}$ are the energies of bands measured with respect to the Fermi level at point $k$, and $g_{k n, k+q m}^{j}$ is the electron-phonon matrix element. The EPC parameter $\lambda$ can be defined as the first reciprocal moment of the spectral function $\alpha^{2} F(\omega)$,

$$
\lambda=2 \int_{0}^{\infty} \frac{\alpha^{2} F(\omega)}{\omega} d \omega \approx \sum_{q j} \lambda_{q j} \omega(q),
$$

where $\omega(q)$ is the weight of a $q$ point in the first BZ. We substituted a Gaussian for the $\delta$ function in Eq. (1.2). The superconducting transition temperature $T_{c}$ has been estimated with the Allen-Dynes modified McMillan equation as ${ }^{32}$

$$
T_{C}=\frac{\omega_{\log }}{1.2} \exp \left[-\frac{1.04(1+\lambda)}{\lambda-\mu^{*}(1+0.62 \lambda)}\right],
$$

where $\omega_{\log }$ is the logarithmic average frequency, and $\mu^{*}$ is the Coulomb pseudopotential. An MP grid of $20 \times 20 \times 20$ was used to ensure $k$-point sampling convergence with Gaussians of width $0.04 \mathrm{Ry}$, in order to approximate the zero-width limit in the calculations of the EPC parameter, $\lambda$.

\section{RESULTS AND DISCUSSIONS}

Structural predictions were performed with the CALYPSO code, considering simulation sizes ranging from one to four $\mathrm{GaH}_{3}$ formula units (f.u.) at 5, 50, 100, 200, and $300 \mathrm{GPa}$. The predicted stable structures were then carefully optimized at a higher level of accuracy at other pressure points for enthalpy curve and electron-phonon coupling calculations. Before the structural searches converged to the most stable structures, we typically generated several hundred structures (e.g., at $300 \mathrm{GPa}$ simulation, we need 100-400 structures to derive the most stable structure of $\operatorname{P} m \overline{3} n$ ). For example, according to our simulations, the structure with the lowest enthalpy at $5 \mathrm{GPa}$ is the base centered monoclinic $C c$ structure ( 2 formula units/unit cell, f.u./cell), and at 50 and 100 GPa, we observed that the simple monoclinic $P 2_{1} / m$ phase (4 f.u./cell) becomes the preferred one [see Figs. 1(a) and 1(b)]. The existence of semimolecular $\mathrm{H}_{2}$ in the $C c$ and $P 2_{1} / m$ structures demonstrates the decomposition trend for $\mathrm{GaH}_{3}$ at low pressure. In addition, two metastable hexagonal phases (6 f.u./cell) with space groups $R \overline{3} c$ and $P 6_{3} 22$, which are very similar to the low-pressure structure of $\mathrm{AlH}_{3}$, built from $\mathrm{AlH}_{6}$ octahedrons linked by Al-H-Al bridges, ${ }^{16}$ are also predicted at $5 \mathrm{GPa}$. Finally, at 200 and $300 \mathrm{GPa}$, a cubic structure of the space group $P m \overline{3} n$ [Fig. 1(c)] is predicted. This structure is characterized by two aluminum atoms located at a simple body centered cubic lattice and equivalent $\mathrm{H}$ atoms forming chains running along each Cartesian direction. Interestingly, the same structure has also been observed in high-pressure $\mathrm{AlH}_{3}$. The nearest $\mathrm{H}-\mathrm{H}$ distance at $160 \mathrm{GPa}$ is $1.547 \AA$, which is much longer than the $\mathrm{H}-\mathrm{H}$ bonding length of $\sim 1.2 \AA$ and shorter

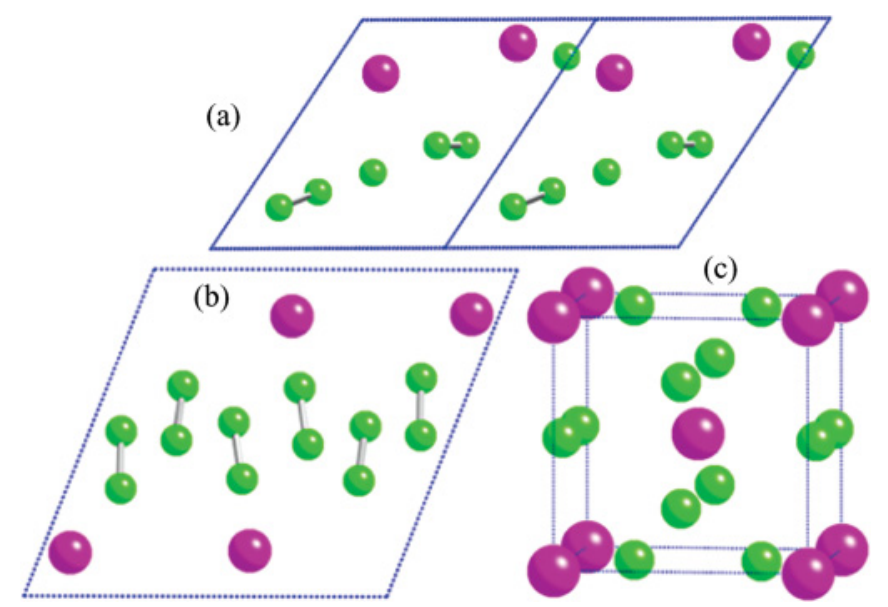

FIG. 1. (Color online) Predicted (a) $C c$, (b) $P 2_{1} / m$, and (c) $P m \overline{3} n$ structures for $\mathrm{GaH}_{3}$. Gallium atoms are shown in pink, and hydrogen atoms are shown in green. The $P m \overline{3} n$ structure contains 2 f.u. for each cell, gallium atoms occupy the crystallographic $2 a(0,0,0)$ site, and hydrogen atoms are located at the $6 c(0.5,0.25,0)$ site.

than the Ga-H distance of $1.73 \AA$. Moreover, $\mathrm{H}-\mathrm{H}$ distances decrease very slowly with increasing pressure and become $1.458 \AA$ at $300 \mathrm{GPa}$, showing that $\mathrm{H}$ atoms do not present any bonding trend.

The enthalpy curves of our predicted structures relative to $R \overline{3} c$ as a function of pressure are presented in Fig. 2. It is clearly seen that below $182 \mathrm{GPa} \mathrm{GaH}_{3}$ is thermodynamically unstable with respect to decomposition of its elements. Actually, $\mathrm{AlH}_{3}$ is also thermodynamically unstable at standard temperature and pressure, and it releases $\mathrm{H}_{2}$ molecules under moderate heating. ${ }^{16,33}$ Above $182 \mathrm{GPa}$, the $\operatorname{Pm} \overline{3} n$ structure clearly becomes the most stable structure. Considering the low mass

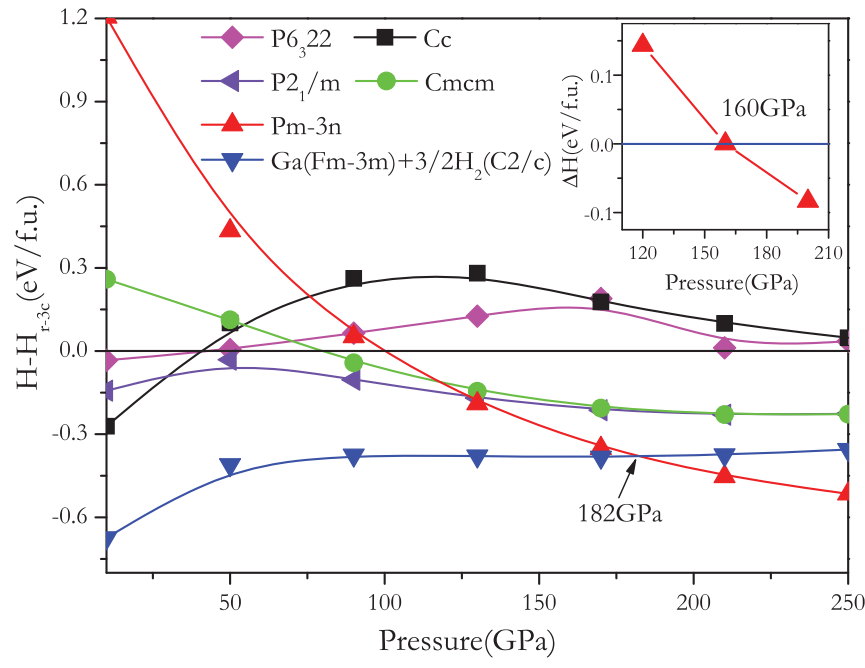

FIG. 2. (Color online) The enthalpies per formula unit of various structures as a function of pressure with respect to $R \overline{3} c$ structure. The decomposition $\left(\mathrm{Ga}+3 / 2 \mathrm{H}_{2}\right)$ enthalpies are calculated by adopting the $C 2 / c$ structure for $\mathrm{H}_{2}{ }^{39}$ and $C m c a, I 4 / m m m, F m \overline{3} m$, and $R \overline{3} c$ structures for $\mathrm{Ga},{ }^{40}$ respectively. Inset: Enthalpies for the $P m \overline{3} n$ structure relative to the decomposition $\left(F m \overline{3} m-\mathrm{Ga}+C 2 / c-\mathrm{H}_{2}\right)$ with the zero-point corrections. 


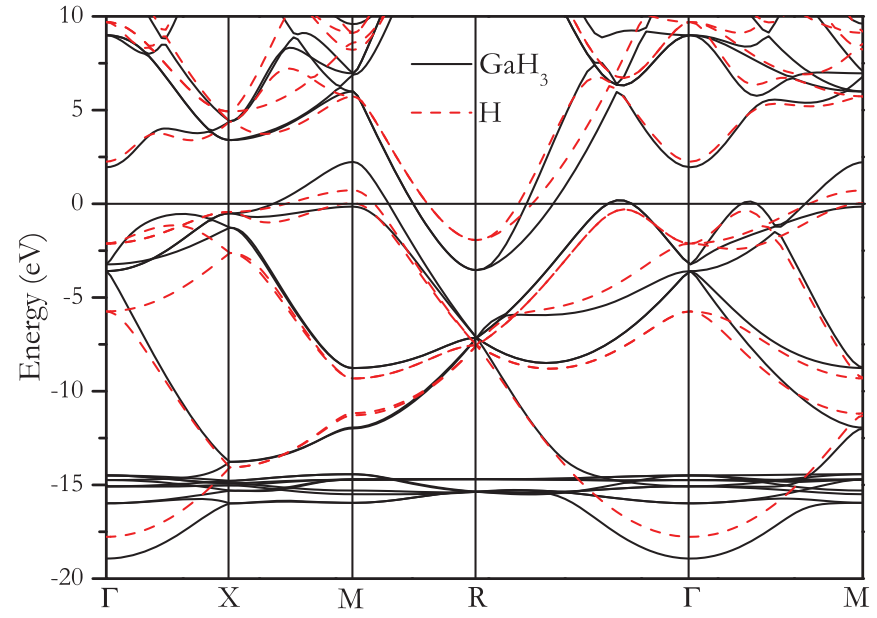

FIG. 3. (Color online) Calculated electronic band structures (full black line) for $\operatorname{Pm} \overline{3} n \mathrm{GaH}_{3}$ at $160 \mathrm{GPa}$ compared with the hypothetical system composed of the hydrogen subsystem immersed in a jellium background (dashed red line).

of $\mathrm{H}$ atoms, we applied the quasiharmonic approximation ${ }^{34}$ to estimate the zero-point ( $\mathrm{ZP})$ energies of $\mathrm{GaH}_{3}, \mathrm{Ga}$, and $\mathrm{H}_{2}$ at 120, 160, and $200 \mathrm{GPa}$. Interestingly, the inclusion of $\mathrm{ZP}$ energies lowers the stabilization pressure of $\operatorname{Pm} \overline{3} n$ to $160 \mathrm{GPa}$.

Motivated by the search for metallic and high superconducting $T_{c}$, hydrogen-rich compounds within the reach of current experimental techniques, the electronic properties, lattice dynamics, and superconductivity of the $P m \overline{3} n$ structure at $160 \mathrm{GPa}$ were also analyzed. Figure 3 presents the calculated electronic band structure for $\operatorname{Pm} \overline{3} n \mathrm{GaH}_{3}$ at $160 \mathrm{GPa}$. The overlap between the conduction and the valence bands under pressure makes $P m \overline{3} n \mathrm{GaH}_{3}$ a metal. As can be seen in the $\Gamma R$ and $\Gamma M$ directions, two maxima in the band structure at $k$-points close to $\Gamma$ are located at the Fermi level, and they make $\mathrm{GaH}_{3}$ have much larger density of states $(\mathrm{DOS})$ at the Fermi energy $\left(3.1 \times 10^{-2}\right.$ states $\left./ \mathrm{eV} / \AA^{3}\right)$ than our calculated data in $\mathrm{AlH}_{3}\left(1.1 \times 10^{-2}\right.$ states $/ \mathrm{eV} / \AA^{3}$ at $80 \mathrm{GPa}$ ).

In order to understand the role of $\mathrm{H}$ atoms in $\mathrm{GaH}_{3}$, we also analyzed the band structure of a hypothetical system composed of only the hydrogen sublattice and a compensating background charge ("hydrogen subsystem"). As can be seen in Fig. 3, the band structures of $\mathrm{GaH}_{3}$ and the hydrogen subsystem are qualitatively similar, which basically indicates that the introduction of Ga atoms does not strongly affect the band structure of the hydrogen sublattice. Therefore, the main role of $\mathrm{Ga}$ atoms in $\mathrm{GaH}_{3}$ is to donate electrons that go to $\mathrm{H}$-like bands, and $\mathrm{Ga}$ associated bands remain unoccupied. The charge transfer from $\mathrm{Ga}$ to $\mathrm{H}$ atoms indicates a strong ionic character of $\mathrm{GaH}_{3}$, similar to those in $\mathrm{AlH}_{3}$ and $\mathrm{LiH}_{n}$. ${ }^{16,35}$ Compared with the behavior observed in $\mathrm{AlH}_{3},{ }^{36}$ near the Fermi energy, the presence of Ga atoms notably shifts the bands down at the $R$ point and slightly up at $M$. In the absence of $\mathrm{Ga}$ atoms, the electronic charge associated to states at $R$ piles up around gallium sites; however, when $\mathrm{Ga}$ atoms are present, the density profile is highly modified. As is shown in Fig. 4, electronic states at $R$ become highly localized around $\mathrm{Ga}$ atoms, and this lowers the energy associated with these states when compared to the hydrogen subsystem, which explains the previously mentioned energy shift in the band structure at $R$. The strong ionic character for $\mathrm{H}$ atoms in $P m \overline{3} n \mathrm{GaH}_{3}$ is different from group IV hydrides (i.e., $\mathrm{SiH}_{4}, \mathrm{GeH}_{4}$, and $\mathrm{SnH}_{4}$ ), where the $\mathrm{H}$ atoms are either bonded to the nearest $\mathrm{H}$ atoms to form semimolecular $\mathrm{H}_{2}$, and/or they are covalently bonded to $\mathrm{M}(\mathrm{M}=\mathrm{Si}, \mathrm{Ge}$, or $\mathrm{Sn})$ atoms to form $\mathrm{M}-\mathrm{H}$ bonds. Interestingly, the high-pressure phase $(P m \overline{3} m)$ of $\mathrm{Si}_{2} \mathrm{H}_{6}$ also has $\mathrm{H}$ atoms with a strong ionic character, which has shown to favor the superconducting transition characterized by a predicted high

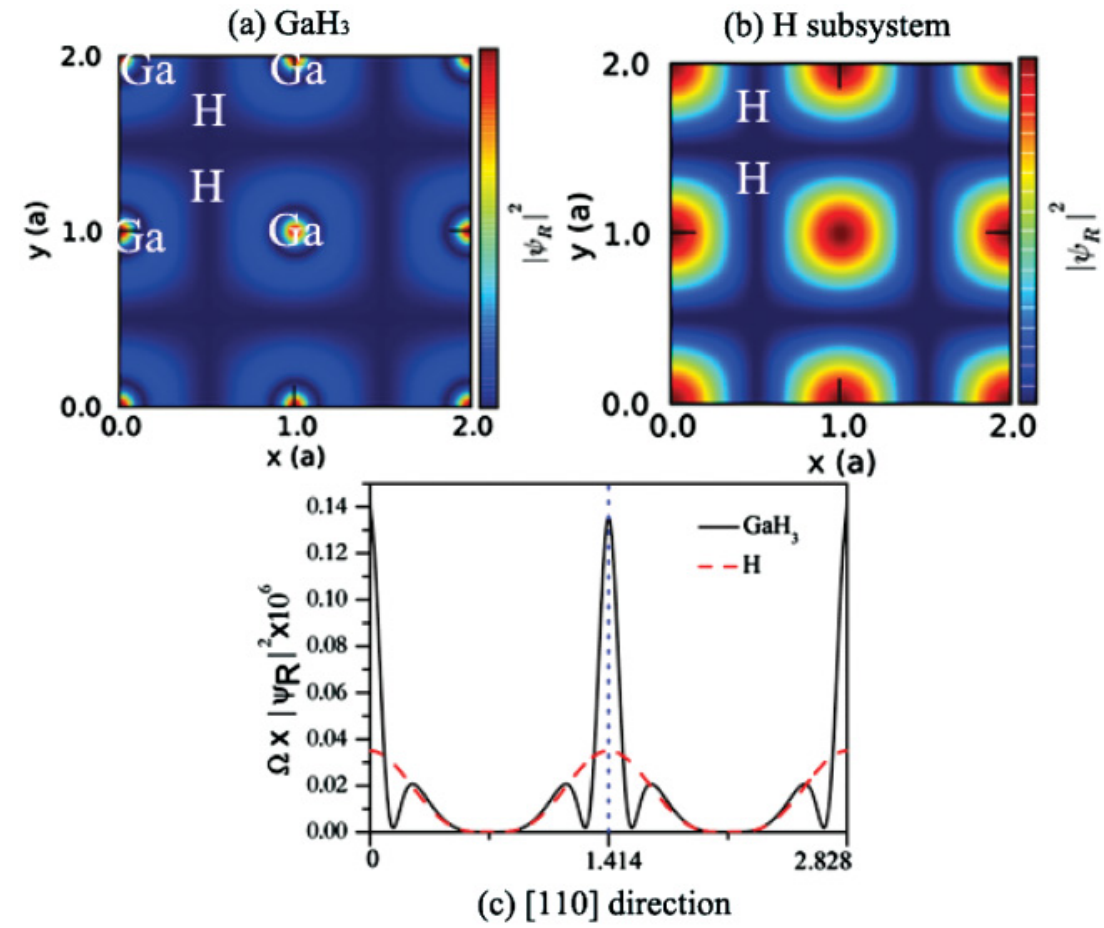

FIG. 4. (Color online) Electronic density contribution from states at the $\mathrm{R}$ point close to the Fermi energy for $P m \overline{3} n \mathrm{GaH}_{3}$ at $160 \mathrm{GPa}$, showing the density on the (100) plane for $\mathrm{GaH}_{3}$ (a) and for the hydrogen system (b). Comparison of the densities in the [110] direction is also shown (c). The vertical lines indicate the positions of the Ga sites. Many periodic repetitions are shown for clarity. 


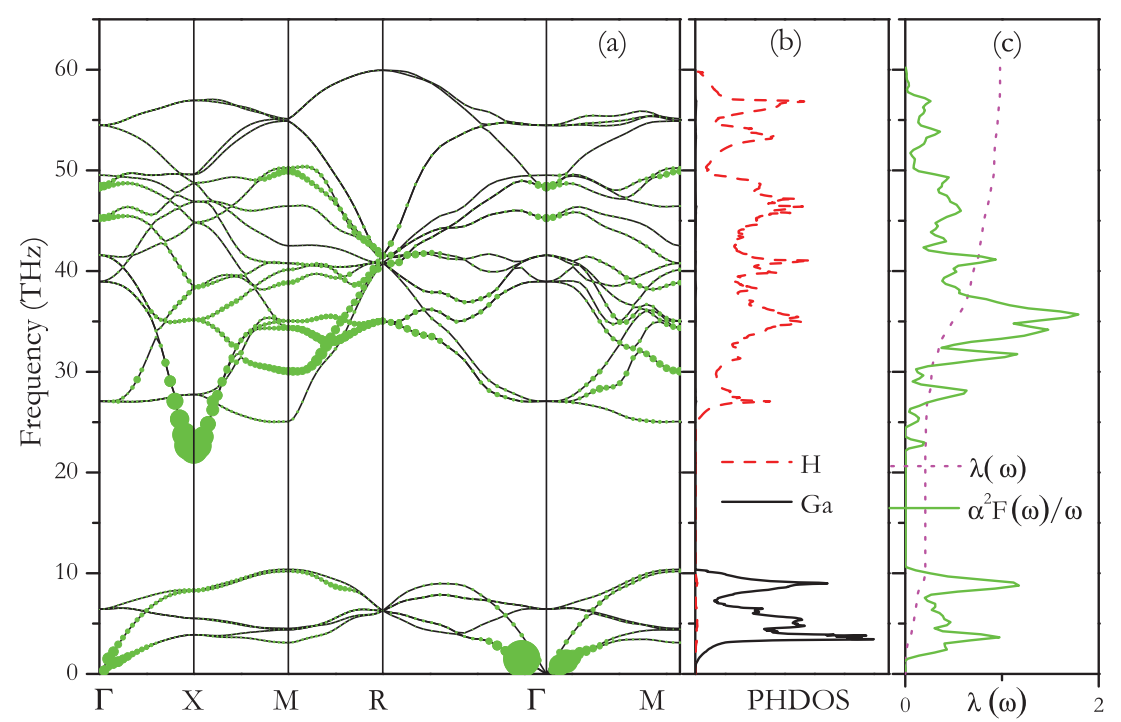

FIG. 5. (Color online) (a) Calculated phonon band structure for $P m \overline{3} n \quad \mathrm{GaH}_{3}$ at $160 \mathrm{GPa}$. Green solid circles show the electron-phonon coupling with the radius proportional to their respective strength. The Eliashberg phonon spectral function, $\alpha^{2} \mathrm{~F}(\omega)$, and the partial electron-phonon integral, $\lambda(\omega)$, in (c) are compared to the phonon DOS projected on $\mathrm{Ga}$ and $\mathrm{H}$ atoms in (b).
$T_{c}$ of $\sim 130 \mathrm{~K}^{37}$ Therefore, we expect that the same trend might also be observed in $P m \overline{3} n \mathrm{GaH}_{3}$. The absence of any imaginary frequency phonon modes [see Fig. 5(a)] proves the dynamical stability of the $P m \overline{3} n$ structure within the studied pressure range. As can be seen in Fig. 5(b), the low-energy phonon modes, with frequencies below $11 \mathrm{THz}$, are mainly associated with $\mathrm{Ga}$ atoms, as expected from their much higher atomic mass, while modes with higher frequencies correspond to $\mathrm{H}$ atoms.

To explore the superconducting properties, we calculated the electron-phonon coupling (EPC) parameter, $\lambda$, the logarithmic average phonon frequency, $\omega_{\mathrm{ln}}$, and the electronic DOS at the Fermi level, $N\left(E_{f}\right)$, at several pressures (Table I). According to our calculations, at $160 \mathrm{GPa}, \lambda$ reaches 0.98 , and $\omega_{\mathrm{ln}}$ is $1271 \mathrm{~K}$, so that the estimated $T_{c}$ becomes $76-83 \mathrm{~K}$, considering typical Coulomb pseudopotential parameters, $\mu^{*}$, of 0.13 and 0.1 , respectively. The large $T_{c}$ in the $P m \overline{3} n$ structure is mainly attributed to the strong electron phonon coupling, $\lambda$, and the high $\omega_{\log }$. In order to understand the origin of this strong $\lambda$, we also calculated the Eliashberg phonon spectral function, $\alpha^{2} F(\omega) / \omega$, and the partial electron-phonon integral, $\lambda(\omega)$. As can be seen in Fig. 5(c), although compressed $\mathrm{AlH}_{3}$ and $\mathrm{GaH}_{3}$ share the same structure, the resulting Eliashberg phonon spectral functions are quite different. Contrary to $\mathrm{AlH}_{3},{ }^{15,17}$ the main contribution to $\lambda$ in $\mathrm{GaH}_{3}$ is not associated with phonons around just two modes at the $X$ point. Similar to the group IVa hydrides, ${ }^{12-14}$ in $\mathrm{GaH}_{3}$ there is an overall contribution of different modes to $\lambda$, and the contribution associated with the previously mentioned two modes around $X$ becomes negligible [Fig. 5(c)]. Therefore, we also expect that the predicted anharmonic renormalization for $\mathrm{AlH}_{3}{ }^{17}$ of these two modes at $X$ will not affect the calculated $T_{c}$ in $\mathrm{GaH}_{3}$. Note that the low-frequency vibrations provide a contribution of $25 \%$ of the total EPC parameter, while the phonon frequencies between $30-40 \mathrm{THz}$ account for nearly $50 \%$ of $\lambda$. To further explore the contribution associated with different phonon modes, solid circles with the radius proportional to the electron-phonon coupling are also plotted in Fig. 5(a). Interestingly, phonon modes between 30 and $36 \mathrm{THz}$ along $M-R$ are primarily responsible for the main peak in the Eliashberg phonon spectral function, $\alpha^{2} F(\omega) / \omega$, and give an important contribution to $\lambda$. Moreover, these phonon modes are mainly associated with the vibrations of ionic $H$. Under pressure, all the phonon modes and the spectral function shift to higher energy, and the major contribution of the intermediate phonon modes to $\lambda$ remains. With increasing pressure, the calculated $\lambda$ and the resulting $T_{c}$ decrease down to $60 \mathrm{~K}$ at $240 \mathrm{GPa}$. However, $\lambda$ is predicted to increase up to 1.19 when pressure decreases to $120 \mathrm{GPa}$, which is the same value as in the Cmca structure for solid hydrogen at $428 \mathrm{GPa}^{38}$ As is shown in Table I, although the calculated $\omega_{\text {ln }}$ lowers with decreasing pressure, reaching $1158 \mathrm{~K}$ at $120 \mathrm{GPa}$, the resulting $T_{c}$ is predicted to increase up to $102 \mathrm{~K}$, and becomes higher than group IV hydrogen-rich compounds.

In summary, theoretical calculations reveal that a metallic cubic structure for $\mathrm{GaH}_{3}$ is stable at high pressures

TABLE I. The calculated phonon frequency logarithmic average $\left(\omega_{\ln }\right)$, EPC parameter $(\lambda)$, the electronic DOS at Fermi level $N\left(E_{f}\right)$, and critical temperature $T_{c}\left(\mu^{*}=0.1\right.$ and 0.13 ) at $120,160,200$, and $240 \mathrm{GPa}$, respectively.

\begin{tabular}{lccrr}
\hline \hline$P(\mathrm{GPa})$ & & & & \multicolumn{2}{c}{$T_{c}(\mathrm{~K})$ with $\mu^{*}=0.1}$, \\
0.13 & $N\left(E_{f}\right)$ states/Spin/Ry/cell & 102 \\
120 & Lambda $(\lambda)$ & $\omega_{\ln }(\mathrm{K})$ & 6.65 & 91 \\
160 & 1.19 & 1158 & 6.13 & 73 \\
200 & 0.98 & 1271 & 5.70 & 72 \\
240 & 0.85 & 1349 & 5.33 & 60 \\
\hline \hline
\end{tabular}


(>160 GPa) against the remarkably high decomposition trend at lower pressures. Electron-phonon coupling calculations show that the $P m \overline{3} n$ phase of $\mathrm{GaH}_{3}$ is superconducting with a high $T_{c}$ of 73-86 K at $160 \mathrm{GPa}$, and even a much higher $T_{c}$ of $102 \mathrm{~K}$ might be expected at $120 \mathrm{GPa}$. Our results demonstrate that hydrogen-rich compounds with $\mathrm{H}$ atoms of ionic character might have a higher $T_{c}$ than those with $\mathrm{H}$ bonded to metal atoms and/or those forming semimolecular $\mathrm{H}_{2}$, supporting the conjecture that hydrogen-rich compounds provide a way to achieve a metallic and superconducting phase at accessible experimental pressures.

\section{ACKNOWLEDGMENTS}

We acknowledge funding support from the National Natural Science Foundation of China (under Grants No. 91022029 and No. 11025418), the research fund of Key Laboratory of Surface Physics and Chemistry (No. SPC201103), and the China 973 Program under Grant No. 2011CB808204. A.B. acknowledges the Department of Education, Universities and Research of the Basque Government, UPV/EHU (Grant No. IT-366-07), and the Spanish Ministry of Science and Innovation (Grant No. FIS2010-19609C02-00). *mym@jlu.edu.cn

${ }^{1}$ N. W. Ashcroft, Phys. Rev. Lett. 21, 1748 (1968).

${ }^{2}$ P. Loubeyre, F. Occelli, and R. LeToullec, Nature 416, 613 (2002).

${ }^{3}$ A. F. Goncharov, E. Gregoryanz, R. J. Hemley, and H. K. Mao,

Proc. Natl. Acad. Sci. USA 98, 14234 (2001).

${ }^{4}$ C. Narayana, H. Luo, J. Orloff, and A. L. Ruoff, Nature 393, 46 (1998).

${ }^{5}$ N. W. Ashcroft, Phys. Rev. Lett. 92, 187002 (2004).

${ }^{6}$ X. J. Chen, V. V. Struzhkin, Y. Song, A. F. Goncharov, M. Ahart, Z. Liu, H.-k. Mao, and R. J. Hemley, Proc. Natl. Acad. Sci. USA 105, 20 (2008).

${ }^{7}$ M. I. Eremets, I. A. Trojan, S. A. Medvedev, J. S. Tse, and Y. Yao, Science 319, 1506 (2008).

${ }^{8}$ M. Hanfland, J. E. Proctor, C. L. Guillaume, O. Degtyareva, and E. Gregoryanz, Phys. Rev. Lett. 106, 095503 (2011).

${ }^{9}$ O. Degtyareva, J. E. Proctor, C. L. Guillaume, E. Gregoryanz, and M. Hanfland, Solid State Commun. 149, 1583 (2009).

${ }^{10}$ M. Martinez-Canales, A. R. Oganov, Y. Ma, Y. Yan, A. O. Lyakhov, and A. Bergara, Phys. Rev. Lett. 102, 087005 (2009).

${ }^{11}$ C. J. Pickard and R. J. Needs, Phys. Rev. Lett. 97, 045504 (2006).

${ }^{12}$ G. Gao, A. R. Oganov, A. Bergara, M. Martinez-Canales, T. Cui, T. Iitaka, Y. Ma, and G. Zou, Phys. Rev. Lett. 101, 107002 (2008).

${ }^{13}$ M. Martinez-Canales, A. Bergara, J. Feng, and W. Grochala, J. Phys. Chem. Solids 67, 2095 (2006).

${ }^{14}$ G. Gao, A. R. Oganov, P. Li, Z. Li, H. Wang, T. Cui, Y. Ma, A. Bergara, A. O. Lyakhov, T. Iitaka, and G. Zou, Proc. Natl. Acad. Sci. USA 107, 1317 (2010).

${ }^{15}$ I. Goncharenko, M. I. Eremets, M. Hanfland, J. S. Tse, M. Amboage, Y. Yao, and I. A. Trojan, Phys. Rev. Lett. 100, 045504 (2008).

${ }^{16}$ C. J. Pickard and R. J. Needs, Phys. Rev. B 76, 144114 (2007).

${ }^{17}$ B. Rousseau and A. Bergara, Phys. Rev. B 82, 104504 (2010).

${ }^{18}$ K. F. Herzfeld, Phys. Rev. 29, 701 (1927).

${ }^{19}$ Y. Wang, J. Lv, L. Zhu, and Y. Ma, Phys. Rev. B 82, 094116 (2010); [http://nlshm-lab.jlu.edu.cn/ calypso.html].

${ }^{20}$ P. Li, G. Gao, Y. Wang, and Y. Ma, J. Phys. Chem. C 114, 21745 (2011).
${ }^{21}$ J. Lv, Y. Wang, L. Zhu, and Y. Ma, Phys. Rev. Lett. 106, 015503 (2011).

${ }^{22}$ L. Zhu, H. Wang, Y. Wang, J. Lv, Y. Ma, Q. Cui, Y. Ma, and G. Zou, Phys. Rev. Lett. 106, 145501 (2011).

${ }^{23}$ J. P. Perdew, K. Burke, and M. Ernzerhof, Phys. Rev. Lett. 77, 3865 (1996).

${ }^{24}$ G. Kresse and J. Furthmüler, Phys. Rev. B 54, 11169 (1996).

${ }^{25}$ P. E. Blöhl, Phys. Rev. B 50, 17953 (1994).

${ }^{26}$ P. Giannozzi, S. Baroni, N. Bonini, M. Calandra, R. Car, C. Cavazzoni, D. Ceresoli, G. L. Chiarotti, M. Cococcioni, I. Dabo, A. D. Corso, S. de Gironcoli, S. Fabris, G. Fratesi, R. Gebauer, U. Gerstmann, C. Gougoussis, A. Kokalj, M. Lazzeri, L. Martin-Samos, N. Marzari, F. Mauri, R. Mazzarello, S. Paolini, A. Pasquarello, L. Paulatto, C. Sbraccia, S. Scandolo, G. Sclauzero, A. P. Seitsonen, A. Smogunov, P. Umari, and R. M. Wentzcovitch, J. Phys. Condens. Matter 21, 395502 (2009).

${ }^{27}$ H. J. Monkhorst and J. D. Pack, Phys. Rev. B 13, 5188 (1976).

${ }^{28}$ S. Baroni, S. de Gironcoli, A. Dal Corso, and P. Giannozzi, Rev. Mod. Phys. 73, 515 (2001).

${ }^{29}$ P. B. Allen and R. Silberglitt, Phys. Rev. B 9, 4733 (1974).

${ }^{30}$ P. B. Allen, Phys. Rev. B 6, 2577 (1972).

${ }^{31}$ J. R. Schrieffer, The Theory of Superconductivity (Benjamin, New York, 1964).

${ }^{32}$ P. B. Allen and R. C. Dynes, Phys. Rev. B 12, 905 (1975).

${ }^{33}$ G. C. Sinke, L. C. Walker, F. L. Oetting, and D. R. Stull, J. Chem. Phys. 47, 2759 (1967).

${ }^{34}$ Y. M. Ma and J. S. Tse, Solid State Commun. 143, 161 (2007).

${ }^{35}$ E. Zurek, R. Hoffmann, N. W. Ashcroft, A. R. Oganov, and A. O. Lyakhov, Proc. Natl. Acad. Sci. USA 106, 17640 (2009).

${ }^{36}$ I. G. Gurtubay, B. Rousseau, and A. Bergara, Phys. Rev. B 82, 085113 (2010)

${ }^{37}$ X. Jin, X. Meng, Z. He, Y. Ma, B. Liu, T. Cui, G. Zou, and H.-k. Mao, Proc. Natl. Acad. Sci. USA 107, 9969 (2010).

${ }^{38}$ L. Zhang, Y. Niu, Q. Li, T. Cui, Y. Wang, Y. Ma, Z. He, and G. Zou, Solid State Commun. 141, 610 (2007).

${ }^{39}$ C. J. Pickard and R. J. Needs, Nature Phys. 3, 473 (2007).

${ }^{40}$ M. I. McMahon and R. J. Nelmes, Chem. Soc. Rev. 35, 943 (2006). 